\title{
Gamma ray bursts monitoring with the ARGO-YBJ experiment in scaler mode
}

\author{
T. Di Girolamo ${ }^{1}$, P. Vallania ${ }^{2}$, and C. Vigorito ${ }^{3}$ \\ ${ }^{1}$ University of Napoli and INFN-Napoli, Complesso Universitario di Monte Sant'Angelo, via Cinthia, 80126 Napoli, Italy \\ ${ }^{2}$ IFSI-Torino, INAF and INFN-Torino, corso Fiume 4,10133 Torino, Italy \\ ${ }^{3}$ University of Torino and INFN Torino, via Pietro Giuria 1, 10125, Torino Italy
}

Received: 26 October 2010 - Revised: 24 January 2011 - Accepted: 25 January 2011 - Published: 4 July 2011

\section{On behalf of the ARGO-YBJ Collaboration}

\begin{abstract}
We report on the search for Gamma Ray Bursts (GRBs) in the energy range $1-100 \mathrm{GeV}$ in coincidence with the prompt emission detected by satellites, using the Astrophysical Radiation Ground-based Observatory at YangBaJing (ARGO-YBJ). With its big active surface $\left(\sim 6700 \mathrm{~m}^{2}\right)$ and large field of view ( $\geq 2 \mathrm{sr}$ ) the ARGO-YBJ air shower detector is particularly suitable to detect unpredictable and short duration events such as GRBs. The search has been performed using the single particle technique in time coincidence with satellite detections both for single events and for the piling up of all the GRBs in time and in phase.

Between November 2004 and June 2010115 GRBs, detected by different satellites (mainly Swift and Fermi), occurred within the field of view of ARGO-YBJ. For 94 of these we searched for a counterpart in the ARGO-YBJ data finding no statistically significant emission. Search methods and results are discussed.
\end{abstract}

\section{Introduction}

The study of GRBs has been carried out mainly from space detecting the primary photons. Due to the fast decrease of the spectrum, the operating energies are usually in the $\mathrm{keV}-$ $\mathrm{MeV}$ range, and only EGRET in the past and now Agile and the Fermi Gamma Ray Space Telescope reached the GeV region, with maximum detectable energies of 30 and $300 \mathrm{GeV}$, respectively. From ground level, the search can be performed by means of large area extensive air shower detectors operating at high altitude, measuring the secondary particles generated by the interaction of the primary photons with the

Correspondence to: C. Vigorito (vigorito@to.infn.it) atmosphere nuclei. This search, which started many years ago (see for example O'Brian and Porter, 1976; Morello et al., 1984; Alexandreas et al., 1994; Castellina et al., 2001; Aglietta et al., 1996) as a particular way to use experiments designed for gamma ray astronomy, requires very stable and reliable detectors. Moreover, at lower energies the number of secondary particles reaching the ground, often only one, does not allow the measurement of the arrival direction, making unfeasible an independent detection.

Forty years after their discovery and more than ten years after the detection of the first afterglow by BeppoSAX, the physical origin of the enigmatic GRBs is still under debate, allowing a great variety of very different models. In these conditions, and mainly in the $>1 \mathrm{GeV}$ energy region, any result could be of great importance to approach the solution of the GRB dilemma.

The sensitivity of ARGO-YBJ may reveal the spectral cutoff in an energy range only partially covered by the satelliteborn detectors and may put constraints on the emission models.

\section{The detector}

The ARGO-YBJ experiment is located at $4300 \mathrm{~m}$ a.s.l. (vertical atmospheric depth $606 \mathrm{~g} \mathrm{~cm}^{2}$ ) at the YangBaJing Cosmic Ray Laboratory $\left(30^{\circ} 06^{\prime} 38^{\prime \prime} \mathrm{N} 90^{\circ} 31^{\prime} 56^{\prime \prime}\right.$ E, Tibet, P.R. of China). The detector is composed of a single layer of Resistive Plate Chambers (RPCs) operated in streamer mode (Aielli et al., 2006) and grouped in 153 units, called clusters, of area $5.7 \times 7.6 \mathrm{~m}^{2}$ each. A cluster is made of $12 \mathrm{RPCs}$ $\left(1.225 \times 2.850 \mathrm{~m}^{2}\right)$ each read by 10 pads $\left(55.6 \times 61.8 \mathrm{~cm}^{2}\right)$ representing the space and time pixels of the array. The clusters are organized in a central full coverage carpet (130 units, $5600 \mathrm{~m}^{2}, 93 \%$ of active surface) enclosed by a guard ring (23 units), which allows the extension of the instrumented area up to $100 \times 110 \mathrm{~m}^{2}$, the increase of the fiducial area and

Published by Copernicus Publications on behalf of the Arbeitsgemeinschaft Extraterrestrische Forschung e.V. 


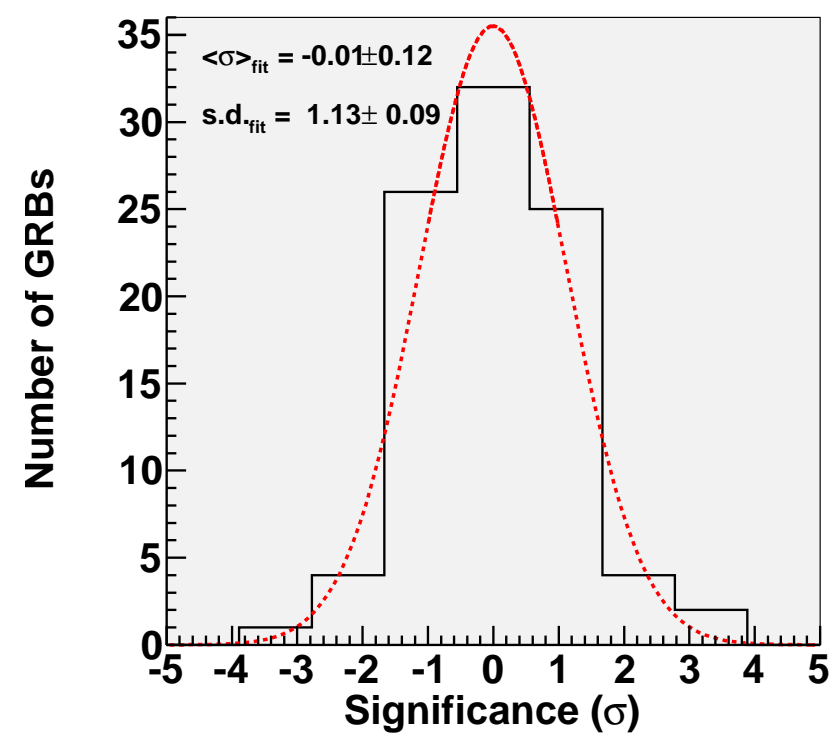

Fig. 1. Distribution of the statistical significances of the set of 94 GRBs with respect to background fluctuations, compared with a Gaussian fit.

the improvement of the accuracy in the core position determination. The detector has two independent data acquisition systems corresponding to the shower and scaler operation modes. In shower mode the arrival time and location of each particle are recorded using the pads allowing the detailed reconstrunction of the shower lateral distribution and the arrival direction. In scaler mode the total counting rate of each cluster is integrated continuously in a $\Delta t=0.5 \mathrm{~s}$ window and recorded for 4 different multiplicity channels $C_{\geq i}$ with $i=1,2,3,4$ (being $150 \mathrm{~ns}$ the coincidence window). The corresponding measured rates are $40 \mathrm{kHz}, 2 \mathrm{kHz}, 300 \mathrm{~Hz}$ and $120 \mathrm{~Hz}$, respectively.

Although this latter technique does not provide information about the energy and arrival direction of the primary cosmic ray, it allows a very low energy threshold of $1 \mathrm{GeV}$ overlapping the highest energy region directly investigated by satellite experiments. Moreover, the use of four different channels sensitive to different energies will provide, in case of positive detection, information on the high energy spectrum slope and possible cutoff (Aielli et al., 2009a).

Since for the GRB search in scaler mode the authentication is only given by the satellite detection, the stability of the detector has to be deeply investigated. Details of this study are widely discussed in Aielli et al. (2008), together with the determination of the effective area, upper limit calculation and expected sensitivity.

\section{GRB monitoring in scaler mode}

The present update collects data from November 2004 (corresponding to the Swift satellite launch) to June 2010, with a detector active area increasing from $\sim 700$ to $\sim 6700 \mathrm{~m}^{2}$. During this period, a total of $115 \mathrm{GRBs}$, selected from the GCN Circulars Archive ${ }^{1}$, was inside the ARGO-YBJ field of view (i.e. with zenith angle $\theta \leq 45^{\circ}$, limited only by atmospheric absorption); for 94 of these gamma ray bursts ARGO-YBJ data were available and they have been investigated by searching for a significant excess in the counting rates coincident with the satellite detection. In order to extract the maximum information from the data, two GRB analyses have been implemented:

- search for a signal from every single GRB;

- search for a signal from the pile-up of all GRBs (stacked analysis).

For both analyses, the first step is the data cleaning and check. For each event, the Poissonian behaviour of the counting rates for multiplicities $\geq 1, \geq 2, \geq 3, \geq 4$ for all the clusters is checked in a period of $\pm 12 \mathrm{~h}$ around the GRB trigger time using the normalized fluctuation function:

$f=(s-b) / \sigma, \quad \sigma=\sqrt{b+b / 20}$.

In this formula, $s$ is the number of counts in a time interval of $10 \mathrm{~s}, b$ the number of counts in $10 \mathrm{~s}$ averaged over a time period of $100 \mathrm{~s}$ before and after the signal, and $\sigma$ the standard deviation, with about 400 independent samples per distribution. The interval of $10 \mathrm{~s}$ has been chosen to avoid any systematic effect caused by environment and instrument (such as atmospheric pressure and detector temperature variations). The expected distribution of $f$ is the standard normal function; all the clusters giving a distribution with measured $\sigma>1.2$ or with anomalous excesses (i.e. n. of entries $>2 \%$ ) in the tail $\sigma>3$ in at least one multiplicity channel are discarded. This guarantees that our data fulfill the requirements on stability and reliability of the detector. In the present running conditions the detector efficiency after the quality cuts is $92 \%$ and the dead time $\leq 1 \%$.

\subsection{Search for single GRBs}

The counting rates of the clusters surviving our quality cuts are added up and the normalized fluctuation function

$f^{\prime}=\left(s^{\prime}-b^{\prime}\right) / \sigma^{\prime}, \quad \sigma^{\prime}=\sqrt{b^{\prime}+b^{\prime} \frac{\Delta t_{90}[s]}{600}}$

is used to give the significance of the coincident on-source counts. In this formula, $s^{\prime}$ is the total number of counts in the $\Delta t_{90}$ time window given by the satellite detector and $b^{\prime}$ the number of counts in a fixed time interval of $300 \mathrm{~s}$ before and after the signal, normalized to the $\Delta t_{90}$ time.

Due to the correlation between the counting rates of different clusters (given by the air shower lateral distribution), the

\footnotetext{
${ }^{1}$ http://gen.gsfe.nasa.gov/gen3_archive.html
} 


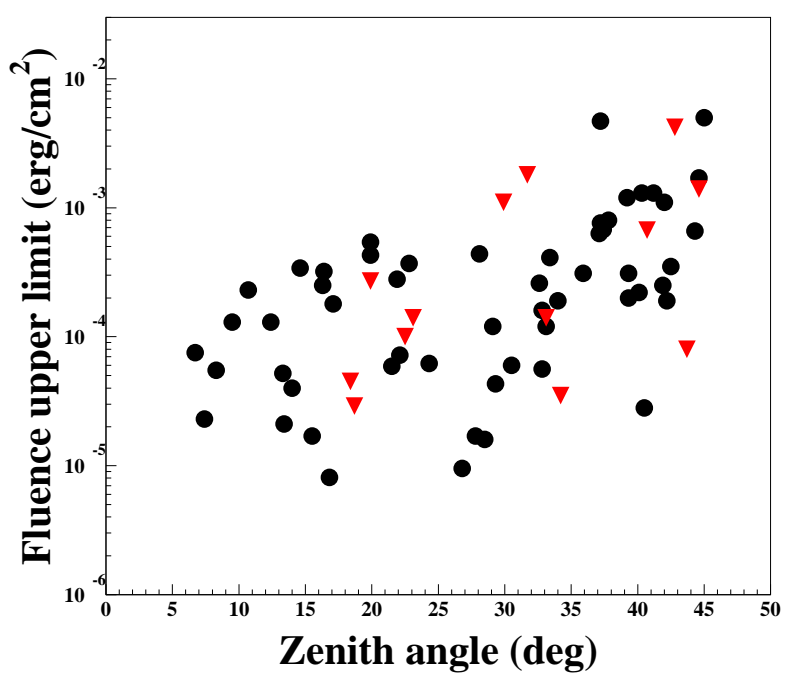

Fig. 2. Fluence upper limits for GRBs with known spectral index as a function of zenith angle. Red triangles for GRBs with know redshift; black dots when $\mathrm{z}=1$ assumed.

distributions of the sum of the counts are larger than Poissonian and this must be taken into account in calculating the significance of a possible signal. The statistical significance of the on-source counts over the background is obtained again in an interval of $\pm 12 \mathrm{~h}$ around the GRB trigger time, using Eq. (17) of $\mathrm{Li}$ and $\mathrm{Ma}$ (1983) (for more details see Aielli et al., 2008). All the results presented here are obtained using the single particle counting rate $\left(C_{1}=C_{\geq 2}-C_{\geq 1}\right)$, corresponding to the minimum primary energy in the ARGOYBJ scaler mode. Figure 1 shows the distribution of the significances for the whole set of 94 GRBs.

No significant excess is found, $3.52 \sigma$ being the maximum significance obtained, with a chance probability of $2.1 \%$ taking into account the total number of GRBs analyzed.

\subsubsection{Fluence upper limits}

With the lack of a positive signal the fluence upper limits are obtained in the $1-100 \mathrm{GeV}$ energy range adopting a power law spectrum and considering the maximum number of counts at 99\% confidence level (c.1.), following Eq. (6) of Helene (1983). For this calculation, two different assumptions are used for the power law spectrum: a) extrapolation from the $\mathrm{keV}-\mathrm{MeV}$ energy region of the spectral index measured by the satellite experiments when available; b) a differential spectral index $\alpha=-2.5$. Since the mean value of spectral indexes measured by EGRET in the GeV energy region is $\alpha=-2.0$ (Dingus et al., 1997), we expect the true upper limits to lie between these two values. For GRBs with known redshift, an exponential cutoff in the spectrum is considered to take into account the effects of extragalactic absorption, which is calculated using the values given in Kneiske et al. (2004).

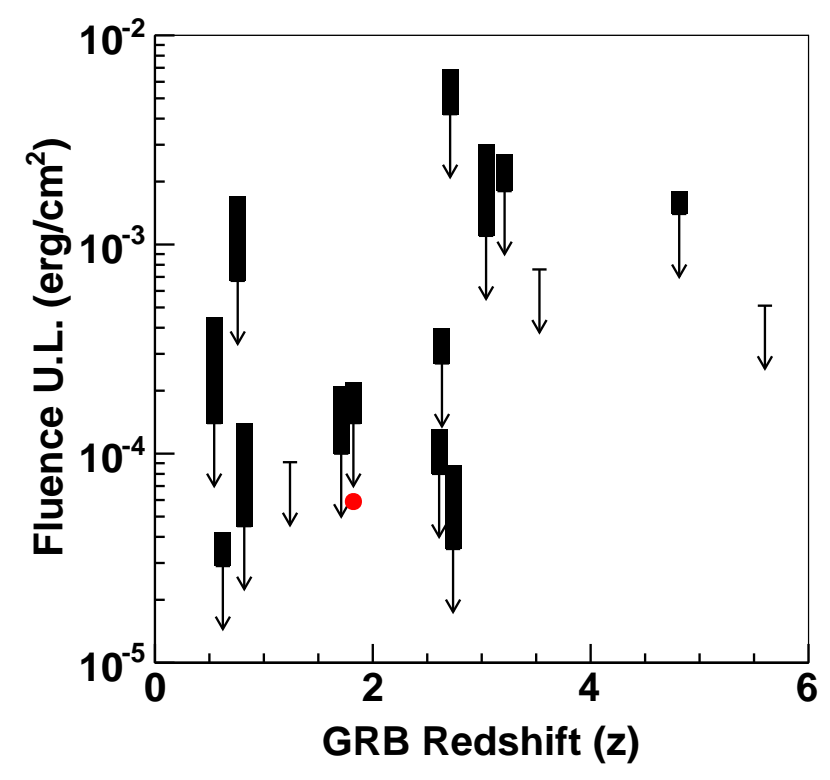

Fig. 3. Fluence upper limits of GRBs as a function of redshift. The rectangles represents the values obtained with differential indexes ranging from the low energy measurement up to $\alpha=-2.5$; the three arrows are the upper limits for this latter case only (GRBs with Cutoff Power Law spectrum; see text for details). The red point shows the integral fluence extrapolated in our sensitivity range for the GRB090902B observed by the LAT instrument on board the Fermi satellite.

For the subset of 68 GRBs with known spectral index $\alpha$, as measured by the satellite experiments, the fluence upper limits have been calculated according to hypothesis a) and they are shown in Fig. 2 as a function of the zenith angle.

For the subset of $16 \mathrm{GRBs}$ with known redshift, the fluence upper limits for the two assumed spectra are shown in Fig. 3. Since the measured low energy differential spectral indexes for these GRBs are always greater than -2.5 , the higher upper limits refer to this extrapolation; for 3 GRBs the measured low energy spectrum is a Cutoff Power Law and only the value obtained assuming $\alpha=-2.5$ is shown. For the other GRBs the rectangles indicate all the upper limits corresponding to differential spectral indexes ranging from the low energy measurement to the fixed value $\alpha=-2.5$.

\subsubsection{Energy cutoff}

The cutoff energy of GRBs is actually unknown. The following procedure is developed in order to determine an upper limit to this energy at least for some GRBs exploting the ARGO-YBJ scaler mode data. When using as the GRB spectrum the extrapolation of the index measured in the keV-MeV region by satellite experiments, the extrapolated fluence is plotted together with our fluence upper limit as a function of the cutoff energy $E_{\text {cut }}$. If the two curves cross in the $2-100 \mathrm{GeV}$ energy range, the intersection gives the upper 


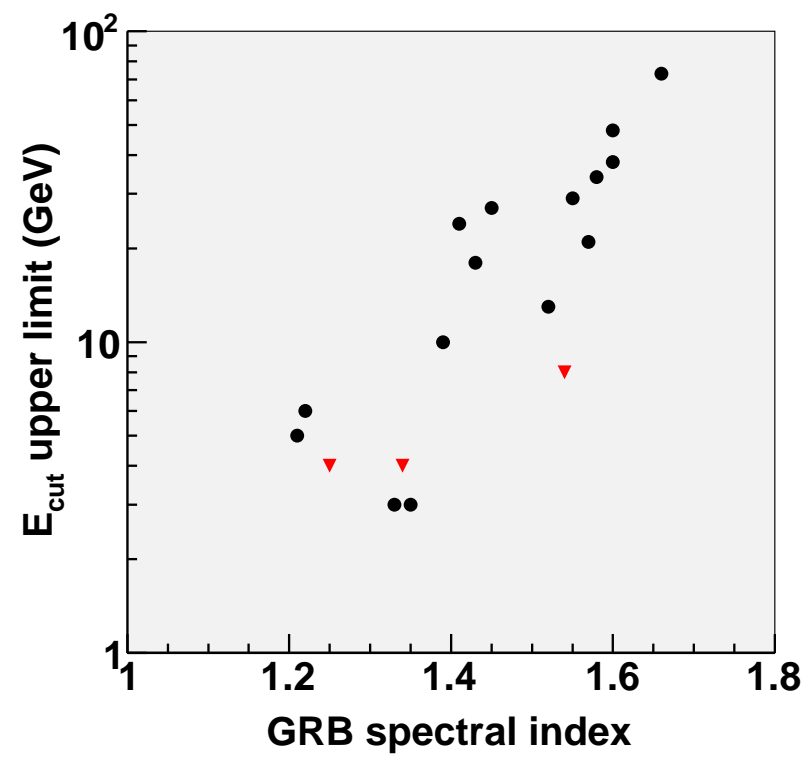

Fig. 4. Cutoff energy upper limits of GRBs as a function of spectral index obtained extrapolating the measured keV-MeV spectra. The values represented by the red triangles are obtained taking into account the extragalactic absorption for the 3 GRBs with known redshift; for the others $z=1$ is adopted.

limit to the cutoff energy. For these GRBs we can state that their spectra do not extend over the obtained $E_{\text {cut }}$ upper limit, with a $99 \%$ c.l. if the spectral index measured by satellites keeps constant. Figure 4 shows the cutoff energy upper limits as a function of the spectral index for the 18 GRBs for which the intersection occurs in the quoted energy range. For 3 of these (red triangles in Fig. 4) the knowledge of the redshift allows the estimation of the extragalactic absorption. When the GRB redshift is unknown a standard value $z=1$ is adopted in the calculation.

\subsection{Pile-up of all GRBs - Stacked analysis}

The search for cumulative effects by stacking all the GRBs either in fixed time durations or in phases of $\Delta t_{90}$ could enhance a possible signal, making it significant, even if the emission of each GRB is below the sensitivity of the ARGOYBJ detector. In this case, less information could be given with respect to the single GRB coincident detection, but we must consider that with the stacked analysis we increase our sensitivity by increasing the number of GRBs, while for the single GRB search we decrease our sensitivity because of the increasing number of trials. On this basis the analysis is performed supposing a common timing feature in all GRBs.

First, all the events during a time window $\Delta t$ (with $\Delta t=$ $0.5,1,2,5,10,20,50,100,200 \mathrm{~s}$ ) after $T_{0}$ (the low energy trigger time given by the satellites) for all the GRBs are added up. This is done in order to search for a possible cumulative high energy emission with a fixed duration after $T_{0}$. A positive observation with a fixed $\Delta t$ could be used as an alternative value to the standard $\Delta t_{90}$ duration. Some indications of such a delayed high energy component has been recently shown for some GRBs by satellite measurements. The resulting overall significance of the GRBs stacked in time with respect to random fluctuations is $-0.70 \sigma$.

A second search is done to test the hypothesis that the high energy emission occurs at a specific phase of the low energy burst, independently of the GRB duration. For this study, all the 79 GRBs with $\Delta t_{90} \geq 5 \mathrm{~s}$ (i.e. belonging to the subset of "long GRB" population, commonly defined by $\Delta t_{90} \geq 2 \mathrm{~s}$ ) have been added up in phase scaling their duration. This choice has been done for both physical and technical reasons, adding up the counts for GRBs of the same class and long enough to allow a phase plot with 10 bins given our time resolution of $0.5 \mathrm{~s}$. There is no evidence of emission at a certain phase, and the overall significance of the GRBs stacked in phase (obtained adding up all the bins) with respect to background fluctuations is $-0.89 \sigma$.

\section{Conclusions}

In this paper we have reported a study concerning the search for $\mathrm{GeV}$ photons from $94 \mathrm{GRBs}$ carried out by the ARGOYBJ air shower detector operated in scaler mode. In the search for $\mathrm{GeV}$ gamma rays in coincidence with the low energy GRBs detected by satellites, no evidence of emission was found for any event. The stacked search, both in time and phase, has shown no deviation from the statistical expectations, therefore excluding any integral effect.

The fluence upper limits obtained in the $1-100 \mathrm{GeV}$ energy range depend on the zenith angle, time duration and spectral index, reaching values down to $10^{-5} \mathrm{erg} \mathrm{cm}^{-2}$. If we consider our sensitivity in terms of expected number of positive detections, our estimate gives a rate between 0.2 and 1 per year, which is comparable with similar evaluations for other experiments working in different energy regions (e.g. Albert et al., 2007).

Finally, the capability of the detector shower mode (here not discussed) to measure the arrival direction and energy of individual showers above a few hundred $\mathrm{GeV}$ allows the ARGO-YBJ experiment to study the GRBs in the whole $1 \mathrm{GeV}-1 \mathrm{TeV}$ range. A detailed discussion on the methods and the first published results can be found in Aielli et al. (2009b).

Edited by: J. Poutanen

Reviewed by: two anonymous referees 


\section{References}

Aglietta, M., et al. (EASTOP collaboration): Search for $\gamma$ ray bursts at photon energies $E_{0} \geq 10 \mathrm{GeV}$ and $E_{0} \geq 80 \mathrm{TeV}$, Astrophys. J., 469, $305-310,1996$.

Aielli, G., et al. (ARGO-YBJ collaboration): Layout and performance of RPCs used in the Argo-YBJ experiment, Nucl. Instr. Meth. A, 562, $92-96,2006$.

Aielli, G., et al. (ARGO-YBJ collaboration): Scaler mode technique for the ARGO-YBJ detector, Astropart. Phys., 30, 85 - 95, 2008.

Aielli, G., et al. (ARGO-YBJ collaboration): Search for Gamma Ray Bursts with th ARGO-YBJ detector in scaler mode, Astrophys. J., 699, 1281 - 1287, 2009a.

Aielli, G., et al. (ARGO-YBJ collaboration): ARGO-YBJ constraints on very high energy emission from GRBs, Astropart. Phys., 32, 47 - 52, 2009b.

Albert, J., et al. (MAGIC collaboration): MAGIC uppper limits on the very high energy emission from Gamma-Ray Bursts, Astrophys. J., 667, 358 - 366, 2007.

Alexandreas, D. E., Allen, G. E., Berkley, D., et al.: Search for ultra-high-energy radiation from gamma-ray bursts, Astrophys. J. 426, 1 - 3, 1994.
Castellina, A., et al. (INCA collaboration): Search for GeV gamma ray bursts at Mount Chacaltaya, in: Proc. 27th ICRC, 2735 2738, 2001.

Dingus, B. L., Catelli, J. R., and Schneid, E. J.: EGRET fluxes and upper limits for energetic gamma ray bursts, in: Proc. 25th ICRC, 3, $33-36,1997$.

Helene, O.: Upper limit of peak area, Nucl. Instr. Meth., 212, 319 322, 1983.

Kneiske, T. M., Bretz, T., Mannheim, K., et al.: Implications of cosmological gamma-ray absorption, Astron. Astrophys., 413, 807 $-815,2004$.

Li, T. and Ma, Y.: Analysis methods for results in gamma-ray astronomy, Astrophys. J., 272, 317 - 327, 1983.

Morello, C., Navarra, G., and Periale, L.: A search for high-energy cosmic gamma-ray bursts, Il Nuovo Cimento, 7C, 682 - 688, 1984.

O'Brian, S. and Porter, N. A.: Upper limits for high energy -rays in association with Vela bursts, Astrophys. Space Science, 42, 73 76, 1976. 